\title{
Ein neuer Zugang zum Konzept des Discounted Cashflow
}

\author{
Lutz Kruschwitz $^{1}$ und Andreas Löffler ${ }^{2}$ \\ erscheint im Heft 1 des Journal für Betriebswirtschaft, 2005
}

\begin{abstract}
A lot of papers have been published about the concept of discounted cash flow or DCF. In this paper we will summarize the recent development and review the main results. After showing the main weaknesses of the concept we will make a sketch of some promising ideas that can form the basis to overcome these weaknesses.
\end{abstract}

\section{Zusammenfassung}

In letzter Zeit sind viele Arbeiten zum Konzept des Discounted Cashflow (DCF) erschienen. In diesem Beitrag wollen wir die wichtigsten Ideen und Ergebnisse referieren. Insbesondere wollen wir darauf eingehen, welche Schwächen die bisher verfolgten Zugänge haben und wie man diese überwinden kann.

JEL-Class. G31, H20

\section{Einführung}

Dass über Unternehmensbewertung wenig geschrieben wird, kann man insbesondere für den deutschen Sprachraum nicht behaupten. Die Zahl der Monografien und Fachaufsätze, die in den letzten zehn Jahren zu diesem Thema verfasst worden sind, ist nur noch mit Mühe zu überschauen. In vielen Beiträgen werden Fragen der Unternehmensbewertung aus der Sicht der praktischen Anwender diskutiert, das sind Wirtschaftsprüfer und Investmentbanker, um die wichtigsten zu nennen. ${ }^{3}$ In diesen Arbeiten geht es nicht darum, neue Modelle zu entwickeln oder Eigenschaften bereits bekannter Modell aus theoretischem Blickwinkel kritisch zu diskutieren. Vielmehr stehen wichtige praktische Probleme im Vordergrund, wie etwa die Folgen von Änderungen des Steuerrechts oder Änderungen von Rechnungslegungsvorschriften. Auch spielen in diesen Arbeiten Fragen der Prognose von Cashflows oder der Bestimmung von Kapitalkosten (etwa mit dem CAPM) eine wichtige Rolle. ${ }^{4}$ Dass man in Arbeiten, die sich an den praktischen Anwender richten oder die gar von Praktikern verfasst werden, im Einzelfall gern zu Ad-hoc-Lösungen greift, darf nicht verwundern.

Den praxisorientierten Beiträgen stehen eher finanzierungstheoretisch geprägte Arbeiten gegenüber. In der fortgeschrittenen angelsächsischen Literatur bedient man sich zum Zweck

\footnotetext{
${ }^{1}$ Freie Universität Berlin, Boltzmannstr. 20, Boltzmannstr. 20,14195 Berlin, e-Mail: LK@wacc.de.

${ }^{2}$ Lehrstuhl für Banken und Finanzierung, Universität Hannover, Königsworther Platz 1, 30167 Hannover, e-Mail: AL@wacc.de. Wir danken dem VVerein zur Förderung der Zusammenarbeit von Lehre und Praxis am Finanzplatz Hannover e.V. für finanzielle Unterstützung.

${ }^{3}$ Siehe Copeland/Koller/Murrin:2000, Mandl/Rabel:1997, Ballwieser:2004 und Peemoeller:2001.

${ }^{4}$ Siehe etwa Hering:1999 oder Stehle:2004.
} 
der Bewertung künftiger Cashflows gern des zeitstetigen Kalküls, der bei Wirtschaftsprüfern oder Investmentbankern auf Grund seines mathematischen Formalismus auf geringe Gegenliebe stößt. ${ }^{5}$ Der bei den Anwendern eher akzeptierte diskrete Kalkül der Unternehmensbewertung wird dagegen von Theoretikern entweder als trivial (man denke an den Fall der ewigen Rente) oder aber als unbequem (man denke an das Binomialmodell) empfunden.

In Monografien, die das Thema Unternehmensbewertung aus finanzwirtschaftlicher Perspektive beleuchten, verwendet man regelmäßig zeitdiskrete Modelle. ${ }^{6}$ Dabei fällt aber auf, dass sich die entsprechende Literatur im wesentlichen auf Erkenntnisse stützt, die in den 60er Jahren gewonnen wurden: die verwendeten Modelle gehen selten oder nie auf moderne finanzierungstheoretische Konzepte wie beispielsweise risikoneutrale Wahrscheinlichkeiten oder gar Martingalmaße ein. Auch die kürzlich entbrannte Diskussion über die Brauchbarkeit von Sicherheitsäquivalenten bei der Unternehmensbewertung bildet hier keine Ausnahme, ${ }^{7}$ da sowohl das Konzept der Nutzenfunktion als auch die Idee des Sicherheitsäquivalents auf Erkenntnisse aus der Mitte des vorigen Jahrhunderts zurückgehen.

Bei der Einbeziehung von Realoptionen in die Bewertungsliteratur handelte es sich dagegen um einen wirklich modernen finanzierungstheoretischen Zugang. ${ }^{8}$ Dennoch müssen wir konstatieren, dass in diesem Gebiet der Abstand zur Praxis enorm ist. Realoptionen galten zur Hochzeit des Neuen Marktes als das Argument, um unverständlich hohe Unternehmenswerte zu begründen. Mit dem Zusammenbruch und anschließenden Verschwinden des Neuen Marktes hat sich auch die Begeisterung für Realoptionen verflüchtigt.

Nun muss man sicher die Frage stellen, warum denn die Theorie der Unternehmensbewertung "modernisiert" werden soll. Welchen Sinn und Zweck soll es haben, moderne Techniken auf ein Gebiet anzuwenden, bei dem praktische Fragestellungen seit jeher die zentrale Rolle spielen? Die Antwort, die wir in diesem Übersichtsartikel geben wollen, ist sehr einfach: Weil man erst durch diese modernen Instrumente der Finanzierungstheorie erkennt, dass der klassische Zugang zur Unternehmensbewertung mit mehreren schwer wiegenden Defiziten belastet ist, die überwunden werden müssen. Um jedoch die Defizite darlegen zu können, müssen wir in einem ersten Abschnitt auf die Grundideen der Unternehmensbewertung eingehen. Die Darstellung der Defizite erfolgt dann in Abschnitt drei.

Es ist für einen Wissenschaftler immer leichter, eine Theorie zu kritisieren, als eine neue Theorie zu entwickeln. Um uns nicht dem Vorwurf auszusetzen, wir wollten nur zerstören, aber nichts konstruktives leisten, wollen wir in diesem Beitrag Überlegungen zu einem Zugang zur Unternehmensbewertung vorstellen, der geeignet sein könnte, die Anwender wie auch die Finanzierungstheoretiker gleichermaßen zu interessieren. Wir glauben, dass unser Konzept die Möglichkeit eröffnet, zum einen interessante theoretische Fragestellungen zu diskutieren, zum anderen aber auch Lösungen für ganz dringende praktische Probleme zu gewinnen. Diese Überlegungen werden wir am anschließenden Abschnitt vier darlegen.

\section{Risiko und Unternehmensbewertung}

Bei der Bewertung eines Unternehmens geht es immer um die Frage, welchen Grenzpreis man für die künftigen Cashflows zahlen sollte, die das Unternehmen abzuwerfen verspricht.

\footnotetext{
${ }^{5}$ Siehe beispielsweise Harris/Pringle:1985, Taggart:1991 oder die Lehrbücher Ingersoll:1987 und Musiela/Rutkowski:1998, um nur einige zu nennen.

${ }^{6}$ Vergleiche beispielsweise Drukarczyk:2003b, Hachmeister:2000, Richter:2002b und Spremann:2002.

${ }^{7}$ Die Diskussion wurde durch die Arbeiten von Schwetzler:2000c und Kuersten:2002 ausgelöst. Sie kann derzeit kaum als abgeschlossen betrachtet werden.

${ }^{8}$ Die Dissertation von Trigeorgis:1986 und seine darauf aufbauenden Arbeiten wurden in der Bewertungsliteratur sehr breit rezipiert. Siehe beispielsweise Amram/Kulatilaka:1999, Copeland/Antikarov:2000, Hommel/Scholich/Vollrath:2001, Koch:1999, Nowak:2003 und Tomaszewski:2000.
} 
Unabhängig davon, ob man solch ein Problem mit den Augen eines Theoretikers oder Praktikers betrachtet, dürfte unbestreitbar sein, dass die künftigen Cashflows unsicher sind. Abstrahieren wir zunächst von der Tatsache, dass das Unternehmen und seine Kapitalgeber Steuern zahlen müssen.

Gehen wir davon aus, dass das zu bewertende Unternehmen im Zeitpunkt $t=1$ freie Cashflows in Höhe von $F C F_{1}$ verspricht. Nehmen wir ferner an, dass die künftigen Zahlungen sicher sind, so können wir ihren heutigen Wert mit Hilfe von Gleichung

$$
V_{0}=\frac{F C F_{1}}{1+r_{f}}
$$

berechnen, wobei $r_{f}$ der risikolose Zinssatz ist. Diese Bewertungsgleichung dürfte so, wie sie hier steht, von jedermann akzeptiert werden, beruht jedoch auf einem wichtigen theoretischen Prinzip der Finanzierungslehre, nämlich dem Prinzip der Arbitragefreiheit. Aufgrund dieser Tatsache lässt sich die Gleichung förmlich beweisen, ohne dass wir unsere Leser mit einem solchen Beweis hier langweilen wollen.

Was ändert sich, wenn wir ins Auge fassen müssen, dass die künftigen Zahlungen unsicher sind? Zunächst einmal ist gar nicht klar, was der Bewerter über die künftigen Zahlungen weiß, wenn er von unsicheren künftigen Cashflows spricht. Kennt er etwa die vollständigen Wahrscheinlichkeitsverteilung der Cashflows oder besitzt er "nur" Kenntnis der Erwartungswerte dieser Zahlungen? Gehen wir - unrealistisch - einmal davon aus, dass der Bewerter die Verteilungen kennt. Dann können wir drei verschiedene Konzepte der Bewertung solcher Zahlungen notieren.

Ein erster Ansatz ist folgender: Sind die künftigen Zahlungen nicht mehr sicher, so könnte man ihren Erwartungswert mit einem Satz diskontieren, der sich aus zwei Komponenten zusammensetzt, dem risikolosen Zinssatz und einer Risikoprämie $p$, also

$$
V_{0}=\frac{E\left[\vec{F} C F_{1}\right]}{1+r_{f}+p} .
$$

Eine derartige Bewertungsgleichung ergibt sich beispielsweise, wenn wir die Gültigkeit des Standard-CAPM voraussetzen, wobei in diesem Fall für die Risikoprämie $p=\left(E[P / p]-r_{f}\right) \beta$ gilt. Es gibt aber auch andere Zugänge zur Bestimmung einer angemessenen Risikoprämie. Es ist üblich, dieses erste Bewertungskonzept als Kapitalkostenkonzept zu bezeichnen. Man darf behaupten, dass dieser Zugang bei der Unternehmensbewertung sehr weit verbreitet ist, ohne Widerspruch befürchten zu müssen.

Eine zweite Alternative nimmt die Risikoadjustierung nicht im Nenner, sondern im Zähler der Bewertungsgleichung vor. Die Bewertungsgleichung nimmt die Form

$$
V_{0}=\frac{\mathrm{CE}}{1+r_{f}}
$$

an, wobei CE für das bedingte Sicherheitsäquivalent steht. Im Rahmen des CAPM wird das Sicherheitsäquivalent mit Hilfe von $E\left[F F^{2} F_{1}\right]-\frac{E\left[P_{m}-r_{f}\right]}{\operatorname{Var}\left[\rho_{m}\right]} \cdot \operatorname{Cov}\left[F_{C F}, P_{m}\right]$ bestimmt, wobei $P_{m}$ die Rendite des Marktportfolios bezeichnet. Jedoch könnte das Sicherheitsäquivalent auch nutzentheoretisch in der Tradition von Bernoulli:1738 ermittelt werden. Zum Konzept des Sicherheitsäquivalents im Rahmen der Unternehmensbewertung hat es in jüngerer Zeit eine heftige Diskussion gegeben. ${ }^{9}$ Die Idee besitzt in der deutschen Literatur zur Unternehmensbewertung einen gewissen Stellenwert, spielt aber in der internationalen Diskussion keine ausgeprägte Rolle.

\footnotetext{
${ }^{9}$ Siehe insbesondere Schwetzler:2000c, Kuersten:2002, Schwetzler:2002a, Diedrich:2003, Wiese:2003, Kuersten:2003b, Kruschwitz/Löffler:2003e.
} 
Ein drittes Konzept bedient sich der Idee der Bewertung mit Arrow-Debreu-Preisen beziehungsweise des risikoneutralen Wahrscheinlichkeitsmaßes $Q$. Unter der Voraussetzung, dass der Kapitalmarkt arbitragefrei ist, gilt nämlich der Fundamentalsatz der Preistheorie, den wir in vorliegendem Zusammenhang in der Form ${ }^{10}$

$$
V_{0}=\frac{E_{Q}\left[F C F_{1}\right]}{1+r_{f}}
$$

zu schreiben hätten. Anstelle der Zahlungen beziehungsweise des Diskontierungssatzes werden nun die Wahrscheinlichkeiten angepasst, mit denen der Erwartungswert der Cashflows gebildet wird. Gerade der letzte Zugang hat sich in der Optionspreistheorie als äußerst fruchtbar erwiesen: nahezu jede Bewertung eines Derivats verwendet den Zugang der risikoneutralen Wahrscheinlichkeiten. Allerdings unterstellt man in der Optionspreistheorie auch, dass die Investoren sehr viel über die Verteilung der zukünftigen Zahlungen (beispielsweise der zugrunde liegende Aktie) wissen. Bei der Unternehmensbewertung wird typischerweise nichts über die Verteilung künftiger Zahlungen eines Unternehmens unterstellt. Stattdessen arbeitet man regelmäßig mit erwarteten Zahlungen. Erwartungswerte im mathematisch-statistischen Sinne sind damit jedoch nicht gemeint; vielmehr handelt es sich um Zahlungen, mit denen der Bewerter aufgrund betriebswirtschaftlicher Planungsüberlegungen rechnet. Insofern ist vorerst nicht erkennbar, wie dieses dritte Konzept für die Ableitung praktikabler Bewertungsgleichungen nutzbar gemacht werden könnte.

Wir haben unsere Überlegungen bisher an einer sehr einfachen Situation illustriert, bei der das Unternehmen nach einer Periode zu existieren aufhört. Unternehmen in der wirklichen Welt leben meistens sehr viel länger. Das bedeutet für die praktische Unternehmensbewertung, dass sie es nicht mit einem Einperiodenmodell, sondern eher mit einem Mehrperiodenmodell zu tun hat. Es stellt sich die Frage, wie vorzugehen ist, um die skizzierten drei Konzepte auf diesen Fall zu übertragen. Wir werden uns dabei auf das Konzept der Risikoprämie konzentrieren.

\section{Defizite in der aktuellen Diskussion über DCF-Verfahren}

Unternehmensbewertung in einer riskanten Welt mit mehreren Perioden wird heutzutage mehr und mehr mit Hilfe von "Discounted Cashflow-Verfahren" vorgenommen. Charakteristisch für diese Verfahren sind folgende Aspekte:

- $\quad$ Es werden zeitdiskrete Modelle diskutiert. Zeitstetige Modelle finden keine Verwendung. ${ }^{11}$

- $\quad$ Die Unternehmen unterliegen einer Unternehmenssteuer. Erst seit kurzem wird auch die Besteuerung der Anteilseigner in den Kalkül einbezogen.

- $\quad$ Die Bewerter wissen nichts oder nur sehr wenig über die stochastischen Eigenschaften der künftigen Cashflows des zu bewertenden Unternehmens.

Wir sind davon überzeugt, dass die DCF-Verfahren, wie man sie gegenwärtig in der Literatur findet, manche theoretische Defizite besitzen. Diese Defizite werden wir jetzt charakterisieren und deutlich machen, wie sie überwunden werden können. Dabei werden wir nicht umhin können, Fragen zu stellen, die auf den ersten Blick recht abstrakt wirken und deren praktische Relevanz sich möglicherweise nicht unmittelbar erschließt. Aber auf den zweiten Blick wird sich zeigen, dass Antworten auf diese Fragen dabei helfen, auch unmittelbar praktische Fragestellungen anzugehen, die sich bisher einer Lösung entzogen haben. Wir glauben, dass sich mit dieser Rekonstruktion der DCF-Verfahren eine Brücke zwischen der reinen Theorie und der praktischen Anwendung durch Wirtschaftsprüfer, Investmentbanker und andere

\footnotetext{
${ }^{10}$ Zum Beweis dieses Zusammenhangs im Mehrperiodenmodell siehe beispielsweise Ross:1978 und Back/Pliska:1991.

${ }^{11}$ Die einzige Ausnahme bilden die drei Arbeiten Harris/Pringle:1985, Taggart:1991 und Löffler:2004.
} 
schlagen lässt.

\section{Erstes Defizit: Kapitalkosten}

In nahezu allen finanzwirtschaftlichen Lehrbüchern, aber wohl auch in jedem Aufsatz zum Thema Unternehmensbewertung findet man den Begriff der Kapitalkosten. Das Konzept der Kapitalkosten ist derart zentral, dass es in den Lehrbüchern als Grundbegriff der Finanzierung meistens bereits auf den ersten Seiten eingeführt wird.

Bemerkenswert ist nun, dass der Kapitalkostenbegriff üblicherweise nur im Rahmen von Einperiodenmodellen präzise definiert wird. Versucht man jedoch, sich darüber Klarheit zu verschaffen, was Kapitalkosten im Zusammenhang mit den bei der Unternehmensbewertung relevanten Mehrperiodenmodellen sein sollen, verliert die im Kontext des Einperiodenmodells vorhandene Präzision rasch ihre klare Kontur. Soweit die begrifflichen Erläuterungen verbal bleiben, lesen wir häufig, dass es sich bei Kapitalkosten um Renditen, um Diskontierungssätze, um Effektivrenditen oder auch um Opportunitätskosten handelt. ${ }^{12}$ In einem Einperiodenmodell (wie beispielsweise dem Standard-CAPM) bedeutet das jeweils dasselbe. Aber im Zusammenhang mit einem Mehrperiodenmodell sind erwartete Renditen nicht unbedingt auch Effektivrenditen; und ebenso kann man sich klarmachen, dass sich Effektivrenditen nicht unbedingt als Diskontierungssätze eignen. In solch einer Situation muss genau geklärt werden, was Kapitalkosten vor dem Hintergrund eines Mehrperiodenmodells sein sollen.

Natürlich haben Wissenschaftler die Freiheit, ihre Begriffe so zu definieren wie sie wollen. Auch in der Finanzierungstheorie gibt es keine richtigen oder falschen Definitionen, sondern nur zweckmäßige und unzweckmäßige. Eine Möglichkeit besteht darin, den Begriff der Kapitalkosten als bedingte erwartete Rendite aufzufassen,

$$
k_{t}:=\frac{E\left[\vec{F} C F_{t+1}+\stackrel{\circ}{V}_{t+1} \mid F_{t}\right]}{\stackrel{\vartheta}{V}_{t}}-1 .
$$

Diese Definition ist von einem theoretischen Standpunkt aus weder schlechter noch besser als jede andere denkbare Alternative. Aus der Sicht praktischer Anwendung hat sie einen großen Vorteil. Wenn Kapitalkosten aus empirischen Daten geschätzt werden müssen, konzentriert man sich immer auf historische Renditen und orientiert sich weder an Effektivrenditen noch an Opportunitätskosten. Zweifellos kann man eine Theorie der Unternehmensbewertung auch auf der Grundlage eines Kapitalkostenbegriffs entwickeln, der sich auf Effektivrenditen bezieht. Es gibt dann aber erhebliche Schwierigkeiten, ein solches Konzept mit empirisch geschätzten Daten zu versorgen. Von daher kann man davon ausgehen, dass die Kapitalkostendefinition im Sinne von Gleichung (1) zweckmäßig ist.

Allerdings darf nicht verhehlt werden, dass mit der Definition (1) Fragen aufgeworfen werden, die bei einer eher qualitativ geprägten Betrachtung unter der Oberfläche bleiben. In Gleichung (1) werden zwei unsichere Größen miteinander ins Verhältnis gesetzt. Ohne weitere Voraussetzungen kann man nicht davon ausgehen, dass das Ergebnis dieser Division eine Zahl und keine Zufallsvariable ist. Mit Zufallsvariablen zu diskontieren, macht aber keinen Sinn, jedenfalls dann nicht, wenn man als Unternehmenswert eine Zahl - und nicht

\footnotetext{
${ }^{12}$ So nutzen beispielsweise [S. 401]Copeland/Weston:1988 den Ausdruck "rate of return" statt "cost of capital”. [S. 20] Brealey/Myers:2000 schreiben explizit, dass "the cost of capital ...is the expected rate of return demanded by investors in common stocks or other securities subject to the same risks as the project”. Auch [S. 43] deMatos:2001 erklärt, dass es sich bei Kapitalkosten um erwartete Renditen handeln soll. Dem gegenüber sprechen [S. 564] Brealey/Myers:2000 von den adjusted cost of capital als geeigneten Diskontierungssätzen. Eine entsprechende Bemerkung gibt es beispielsweise auch bei [S. 722] Miles/Ezzell:1980, wo es heißt, dass "at any time $k, \rho$ is the appropriate rate for discounting the time $i$ expected unlevered cash flow in period $j$ where $\rho$ is referred to as the unlevered cost of capital”. Spremann:2002 definiert dagegen Kapitalkosten als Effektivrenditen.
} 
auch wieder eine Zufallsvariable - erhalten will.

Dieses Problem lässt sich nur mit Hilfe einer heroischen Annahme überwinden. Man muss voraussetzen, dass die Kapitalkosten im Sinne der Definition von Gleichung (1) keine Zufallsvariablen sind, sondern vielmehr Zahlen darstellen. Dann und nur dann sind wir in der Lage, den von einigen Lesern vielleicht als selbstverständlich empfundenen Sachverhalt zu beweisen, dass die Diskontierung künftiger Cashflows mit Kapitalkosten auf Unternehmenswerte führt,

$$
\stackrel{\ominus}{V}_{t}=\sum_{s=t+1}^{T} \frac{E\left[\mathcal{F C F}_{s} \mid F_{t}\right]}{\left(1+k_{t+1}\right) \cdot \ldots \cdot\left(1+k_{s}\right)} .
$$

Es sei betont, dass diese heroische Annahme notwendig für die Bestimmung von Unternehmenswerten nach (2) ist. ${ }^{13}$

\section{Zweites Defizit: Kapitalstrukturen}

Unterliegt das zu bewertende Unternehmen der Besteuerung, so genießt es Vorteile, wenn es sich verschuldet, da die Kreditzinsen im allgemeinen von der Bemessungsgrundlage der Unternehmenssteuer abgezogen werden dürfen. Die Steuervorteile sind daher um so größer, je stärker das Unternehmen verschuldet ist. Bei der Anwendung von Bewertungsgleichungen ist es daher wichtig, die gegenwärtige und künftige Verschuldung des Unternehmens korrekt zu messen.

Traditionelle DCF-Verfahren verlangen dabei, dass sowohl das Fremdkapital als auch das Eigenkapital des Unternehmens in Marktwerten gemessen werden. Bei der praktischen Unternehmensbewertung wird gegen dieses Prinzip nur allzu häufig verstoßen. Die Messung erfolgt nicht in Marktwerten, sondern - unkorrekt - in Buch- oder Bilanzwerten. Das mag dort, wo es nur auf die absoluten Werte des Fremdkapitals ankommt, gerade noch hingehen, weil man zeigen kann, dass Buch- und Marktwerte beim Fremdkapital meistens nahe beieinander liegen. Für das Eigenkapital gilt das zumindest bei florierenden Unternehmen aber ganz und gar nicht. Und wenn dann in den Bewertungsgleichungen Fremdkapitalquoten beziehungsweise Verschuldungsgrade eine Rolle spielen, so kann es zu gravierenden Fehlbewertungen führen, wenn diese auf der Basis von Buch- anstelle von Marktwerten ermittelt wurden.

Im Rahmen des insbesondere im angelsächsischen Sprachraum verbreiteten WACCKonzeptes spielen solche Fremdkapitalquoten eine zentrale Rolle. Man kann sicher sein, dass Manager, welche nach den künftigen Fremdkapitalquoten des von ihnen geführten Unternehmens gefragt werden und beispielsweise antworten, dass sie die Absicht hätten, die Fremdkapitalquote innerhalb der nächsten $n$ Jahre zu halbieren, in Buchwerten denken und ganz verwundert wären, wenn man ihnen klar zu machen versuchte, dass sie ihre Überlegungen in Marktwerte übersetzen müssen. Ein Manager, der eine in Marktwerten gemessene Fremdkapitalquote beibehalten will, müsste entschlossen sein, die Hälfte der Unternehmensschulden zurückzuzahlen, wenn der Aktienkurs um 50\% fällt. Dass ein solches Prinzip von verantwortlichen Managern tatsächlich verfolgt wird, wenn sie erklären, die Fremdkapitalquote konstant halten zu wollen, darf wohl kaum angenommen werden. ${ }^{14}$

Sehr erstaunlich ist nun, dass es in der theoretischen Literatur so gut wie keine Versuche gibt, konsistente Bewertungsgleichungen für den Fall zu entwickeln, dass Fremdkapitalquoten so gemessen werden, wie Praktiker dies gern tun, nämlich in Buchwerten. Ebenso erstaunlich ist

\footnotetext{
${ }^{13}$ Siehe dazu Laitenberger/Löffler:2002 sowie Laitenberger:2004. Wilhelm:2004 dagegen unterstellt, dass die Kapitalkosten voneinander unabhängig sind, um dann mit der Definition $E\left[\frac{1}{1+k_{t}} \oint^{\prime}=: \frac{1}{1+k_{t}}\right.$ zu arbeiten.

${ }^{14}$ Siehe dazu [S. 717]Schildbach:2000.
} 
es, dass die Praxis nicht vernehmlich nach solchen Bewertungsgleichungen ruft. Stattdessen gibt man sich mit Bewertungsgleichungen zufrieden, in die die entsprechenden Kennzahlen zu Marktwerten einfließen müssen und misst diese notfalls - mit mehr oder weniger schlechtem Gewissen - falsch.

Die in diesem Zusammenhang häufig zu hörende Beschwichtigung, dass der Bewertungsfehler auf Grund niedriger Steuersätze klein sei, ist unbegründet. Wenn man nicht einmal weiß, wie der richtige Unternehmenswert zu Stande kommt, wie kann man dann überzeugt sein, dass dieser Wert sich von einem falsch berechneten nur geringfügig unterscheidet? Eine Aussage zur Größenordnung des Bewertungsfehlers kann man sich erst leisten, wenn man im Besitz der korrekten Bewertungsgleichung ist.

\section{Drittes Defizit: Ad-hoc-Aktualisierungen}

Ein Unternehmen, das anteilig fremdfinanziert ist, genießt Steuervorteile. Wie hoch diese Steuervorteile sind, hängt von mehreren Umständen ab, zum Beispiel von der Finanzierungspolitik, die das zu bewertende Unternehmen betreibt. Von erheblichem Einfluss ist aber auch das Steuerregime, dem das betrachtete Unternehmen und seine Financiers unterliegen.

Die grundlegenden theoretischen Beiträge zur Bewertung von Unternehmen mit Hilfe von DCF-Verfahren beruhen auf der Annahme, dass man es mit einem wenig komplexen Steuersystem zu tun hat. Zumeist ist unterstellt worden, dass lediglich auf Firmenebene Steuern erhoben werden und Steuern auf der Ebene der Kapitalgeber unbeachtet bleiben können. Im deutschen Sprachraum vertritt man demgegenüber seit langer Zeit die Ansicht, dass die Steuern auf der persönlichen Ebene keinesfalls vernachlässigt werden dürfen. ${ }^{15}$

Aus diesem und dem weiteren Grunde, dass die Steuertarife und Besteuerungsgrundsätze vom Gesetzgeber ständig verändert werden, ist es erforderlich, immer wieder darüber nachzudenken, wie Bewertungsgleichungen an neue Steuerregime angepasst werden müssen.

Der Theoretiker geht dabei andere Wege als der unter Zeit- und Erfolgsdruck stehende Praktiker. Wenn ein Theoretiker eine neue Bewertungsgleichung entwickelt, muss er die (neuen steuerlichen) Annahmen explizieren, von denen er ausgeht, und mit Hilfe von logischen Operationen nachweisen, dass die von ihm entworfene Bewertungsgleichung korrekt ist. Diese Operationen können von Dritten überprüft und eindeutig als wahr oder falsch entschieden werden. Die Empfehlungen von Praktikern gehorchen anderen Gesetzen. Bewertungsgleichungen, die aus ihrer Feder stammen, müssen "praktikabel” und "plausibel” sein. Mit langwierigen mathematischen Herleitungen und Beweisen können oder wollen sich die Praktiker nicht aufhalten. Aus theoretischer Sicht sind deren Vorschläge aber meistens nur ad hoc und sie bergen daher die Gefahr, dass sie logisch in sich widersprüchlich sind.

Ein drastisches Beispiel bildet die auf den ersten Blick selbstverständliche Einbeziehung einer Steuer auf Anteilseignerebene im Fall riskanter Investitionen durch das folgende ad hoc Vorgehen: Man passt die Kapitalwertgleichung dahingehend an, dass im Zähler statt der

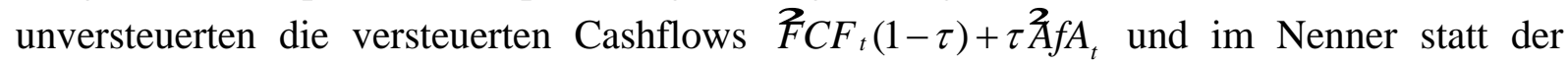
unversteuerten Kapitalkosten $k$ die versteuerten Kapitalkosten $k(1-\tau)$ verwendet werden. ${ }^{16}$ Obwohl dieses Vorgehen "intuitiv einleuchtet”, kann es in nicht unrealistischen Fällen zu Arbitragegelegenheiten führen. Die Multiplikation der Kapitalkosten mit dem Term 1- $\tau$ setzt nämlich voraus, dass die Einkommensteuer nicht den Zufluss, sondern den ökonomischen Gewinn besteuert. Das Steuersystem wird damit inkonsistent. Es besteuert die Finanzanlage anders als die Sachinvestition. Selbst bei Unternehmen, die keine Kursgewinne

\footnotetext{
${ }^{15}$ Vergleiche dazu [Abschnitt A, Tz 224 f.]WPH:2002.

${ }^{16}$ Dieser Vorschlag findet sich nicht nur im deutschen Sprachraum (wieder [Abschnitt A, Tz 224 f.]WPH:2002), sondern auch in angelsächsischen Lehrbüchern, wie etwa [S. 411]Copeland/Koller/Murrin:2000.
} 
aufweisen, lässt sich aus dieser Inkonsistenz eine Arbitrage erzeugen. ${ }^{17}$

Wir wollen uns nicht damit begnügen, die Defizite der DCF-Theorie aufzuzeigen. Als Theoretiker haben wir auch die Pflicht, diese Defizite zu überwinden und Ideen zu formulieren, wie die Theorie weiter entwickelt und verbessert werden kann. Dabei wollen wir uns an geeignete Leitlinien halten, die wir im nächsten Abschnitt darlegen werden.

\section{Eine neue Theorie des Discounted Cashflow}

Neue Theorien entstehen nicht im luftleeren Raum. Sie werden in der Absicht entwickelt, Schwächen bereits vorhandener Ansätze zu überwinden und neue Ergebnisse zu erzielen. An dieser Stelle sollen die Grundlinien einer neuen Theorie des Discounted Cashflow entworfen werden. ${ }^{18}$ Die Vorgehensweise, derer wir uns bedienen wollen, charakterisieren wir am besten dadurch, dass wir zentrale Leitlinien herausarbeiten. Diese Leitlinien sind folgende.

1. Jede Theorie benötigt Annahmen. Das ist auch bei der Theorie des Discounted Cashflow nicht anders. Die Annahmen sollen logisch notwendig, widerspruchsfrei sowie zweckmäßig sein. Es muss geprüft werden können, ob und inwieweit sie empirisch zutreffend sind.

Wir werden wichtige Annahmen im Folgenden genauer beschreiben und auch darlegen, warum sie zweckmäßig sind. Wir wollen jedoch nicht darüber diskutieren, welche der Annahmen besonders realitätsnah sind. Auf eine empirische Analyse wird gänzlich verzichtet.

So könnte man es sich beispielsweise zur Aufgabe machen, die in der Praxis am häufigsten verwendete Finanzierungspolitik herauszuarbeiten und ausschließlich für diese Politik eine Bewertungstheorie zu entwickeln. Das entspricht aber nicht unserem Vorstellungen. Wir halten es vielmehr zunächst für erforderlich, alle nur denkbaren Finanzierungspolitiken zu betrachten und für jede, soweit möglich, eine passende Bewertungsgleichung zu beweisen.

2. In der Finanzierungstheorie und daher auch in der entsprechend geprägten Theorie der Unternehmensbewertung gibt es zwei miteinander konkurrierende Paradigmen: die Arbitragefreiheit und das Gleichgewicht. Es ist unbestritten, dass das Gleichgewicht das stärker einschränkende Konzept ist: In jedem Gleichgewicht herrscht Arbitragefreiheit, aber ein arbitragefreier Markt muss nicht notwendig im Gleichgewicht sein. Daher empfiehlt es sich, ausschließlich von der Voraussetzung eines arbitragefreien Marktes auszugehen. Das hat mehrere Vorteile.

Um ein Gleichgewicht zu charakterisieren, müssen Investoren mit ihren Erstausstattungen und Nutzenfunktionen beschrieben werden. Obwohl die Theorie hier beachtliches geleistet hat, sind wir von einer empirischen Bestimmung individueller Nutzenfunktionen noch weit entfernt. Das gilt im Übrigen auch für die im Gleichgewicht notwendigen Existenz- und Eindeutigkeitsbeweise, die in der Literatur gern unterschlagen werden. Mit diesen Schwierigkeiten hat man nicht zu kämpfen, wenn man sich auf die Voraussetzung der Arbitragefreiheit beschränkt.

Es gibt noch ein weiteres Argument dafür, sich auf das Prinzip der Arbitragefreiheit zurückzuziehen. Das Paradigma des Gleichgewichts wird, wie auch die Ideen der Informationsökonomie und des rationalen Verhaltens, selbst in der ökonomischen Theorie verhältnismäßig stark kritisiert. Es gibt viele Arbeiten, die gute Argumente dafür vortragen, warum Märkte im Ungleichgewicht sind, weshalb Vertragsparteien nicht identisch informiert sind und weshalb sich Investoren nicht rational verhalten. All diese Überlegungen waren in der Vergangenheit Ausgangspunkt für interessante theoretische Entwicklungen. Es hat aber keinen einzigen Versuch gegeben, eine

\footnotetext{
${ }^{17}$ Vergleiche zu Einzelheiten Kruschwitz/Löffler:2004b.

${ }^{18}$ Vergleiche dazu Kruschwitz/Löffler:2003a.
} 
Theorie nicht-arbitragefreier Märkte zu erarbeiten. Und es ist auch völlig klar warum: weil andauernde Arbitragegelegenheiten empirisch nicht beobachtet werden und ein Markt, der stets und ständig Arbitragegelegenheiten bietet, darüber hinaus theoretisch ganz und gar uninteressant ist. Das Prinzip der Arbitragefreiheit ist eines der wenigen unbestrittenen Paradigmen der Ökonomie.

3. Das Thema Unternehmensbewertung hat sich immer im Spannungsfeld zwischen Theorie und Praxis bewegt und wird das auch künftig tun. Aus diesem Grund muss man verlangen, dass die Ergebnisse einer Theorie der Unternehmensbewertung immer auch umsetzbar bleiben. Es ist nun eine Tatsache, dass bei der Anwendung der DCFTheorie Praktiker im Allgemeinen durchaus klare Aussagen zu den Erwartungswerten zukünftiger Cashflows machen können, aber nahezu nichts über die Wahrscheinlichkeitsverteilungen dieser Cashflows aussagen können. Eine DCFTheorie muss darauf Rücksicht nehmen.

Die Unfähigkeit der Praktiker, Vorstellungen über die Wahrscheinlichkeitsverteilungen künftiger Cashflows entwickeln zu können, hat Konsequenzen, die man nicht auf den ersten Blick erkennt. Der Kern unserer Idee für eine neue DCF-Theorie besteht darin, Techniken aus der Optionspreistheorie auf die Unternehmensbewertung zu übertragen.

Dort allerdings wird unterstellt, dass die stochastischen Prozesse, denen die Preise der underlying assets folgen, sehr genau bekannt sind. Das aber steht im drastischen Widerspruch zu der gerade skizzierten Leitlinie, über die Verteilungen künftiger Zahlungen gerade nichts zu wissen. Auch hier kommt man aber weiter, wenn man von der Voraussetzung schwach auto-regressiver Cashflows ausgeht, ${ }^{19}$

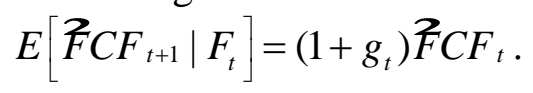

Ohne nähere Erläuterung bleibt das unverständlich. Deshalb wollen wir auf die Annahme (3) der schwachen Auto-regression etwas genauer eingehen.

Zuerst fällt ein wichtiger Unterschied zu den aus der Statistik wohlbekannten AR(1)Prozessen auf. Während dort die Wachstumsraten zeitunabhängig sind, kann hier der Parameter $g_{t}$ zeitlich veränderlich sein. Das führt zu größeren Schwierigkeiten bei der empirischen Validierung des Modells, erweitert aber den Anwendungsbereich wegen der höheren Allgemeinheit erheblich.

Auch Praktiker, die auf eine Charakterisierung der Cashflow-Verteilungen verzichten wollen, können sicherlich mit der Annahme (3) konform gehen. Man kann nämlich zeigen, dass es bei einer gegebenen Folge erwarteter Cashflows immer ein zu Grunde liegendes Binomialmodell gibt, dass der Gleichung (3) genügt. Wer den Verteilungen nicht auf den Grund gehen will, kann ihre Existenz also immer voraussetzen.

Sind die Cashflow nun im obigen Sinne schwach auto-regressiv, dann lässt sich eine Aussage beweisen, die bereits bei der Diskussion der Kapitalkosten angedeutet wurde. Dazu konzentrieren wir uns auf Abbildung 1.

Wir hatten bereits darauf hingewiesen, dass man dem Begriff der Kapitalkosten verschiedene Bedeutungen zuweisen kann, die alle miteinander übereinstimmen, wenn man es mit einem Einperiodenmodell zu tun hat. Die Abbildung 1 macht nun deutlich, dass in einem Mehrperiodenmodell die Dinge anders liegen. Nehmen wir dazu an, dass die erwarteten Renditen eines Unternehmens zeitlich konstant wären, wir bezeichnen sie mit $k$. Betrachten wir weiter den Cashflow $F C F_{s}$, der im Zeitpunkt $s$ fällig wird und stellen die Frage, welchen Wert dieser Cashflow im Zeitpunkt $t<s$ besitzt. Aus der Theorie der Arbitragefreiheit folgt nun sofort, dass dieser Wert genau dem Ausdruck

\footnotetext{
${ }^{19}$ Diese Annahme findet sich in der Literatur beispielsweise bei Garman/Ohlson:1981 und Feltham/Ohlson:1995.
} 


$$
\frac{E_{Q}\left[{ }_{F} C F_{s} \mid F_{t}\right]}{\left(1+r_{f}\right)^{s-t}}
$$

entsprechen muss. Es ist aber keinesfalls offensichtlich, dass man diesen Wert auch mittels der Rechenregeln des Barwertkalküls bestimmen kann, dass also auch

$$
\frac{E_{Q}\left[\mathrm{~F}_{\mathrm{F}} \mathrm{F} F_{s} \mid F_{t}\right]}{\left(1+r_{f}\right)^{s-t}}=\frac{E\left[\mathrm{~F} C F_{s} \mid F_{t}\right]}{(1+k)^{s-t}}
$$

gilt. Auf der rechten Seite der Gleichung handelt es sich ja nicht um einen Diskontierungssatz eines einzelnen Cashflows: Vielmehr stellt die Größe $k$ definitionsgemäß eine erwartete Rendite des gesamten Unternehmens dar. Eins der wichtigsten Ergebnisse ist nun die Aussage, dass im Fall schwach auto-regressiver Cashflows der Zusammenhang (4) beweisbar wird. ${ }^{20}$

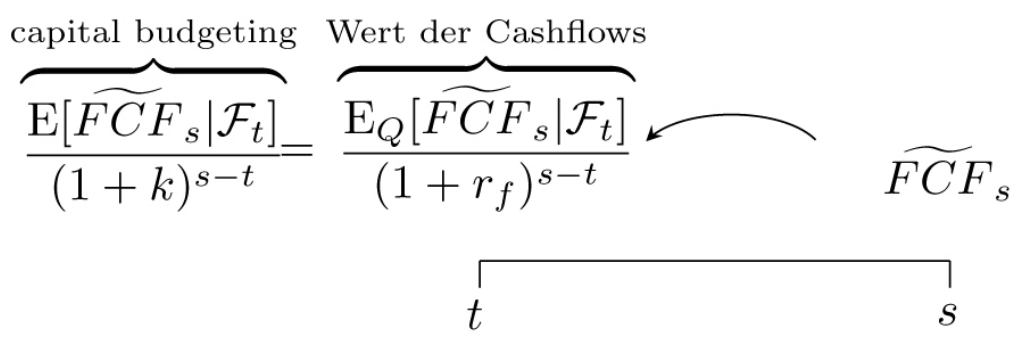

Abbildung 1. Kapitalkosten sind Diskontierungssätze

Neben den theoretischen Leitlinien wollen wir noch einen Aspekt ansprechen, der eher didaktischen Charakter hat. Eine neue Theorie der Unternehmensbewertung muss nicht nur bisher unbekannte Erkenntnisse erlauben, sie sollte vielmehr nach Möglichkeit auch verständlich vermittelt werden können. Ein nicht unbedingt ermutigendes Beispiel stellen die zeitstetigen Theorien dar, bei denen Anschluss an die aktuelle Forschung nur mit einem hohen formalen Aufwand hergestellt werden kann. Aber auch die zeitdiskreten Ansätze verlangen, wenn sie vollständig entwickelt werden, eine Beschäftigung mit $\sigma$-Algebren, Wahrscheinlichkeitsmaßen, einer fortgeschrittenen Integrationstheorie sowie der Auseinandersetzung mit bedingten Erwartungswerten. ${ }^{21}$ Bei der Präsentation des neuen Konzepts müsste versucht werden, hier einen eleganten Mittelweg zu beschreiten. Statt einer aufwendigen mathematischen Einführung (die im Übrigen keine weitere Verwendung finden würde) sollte man sich auf elementare Rechenregeln für bedingte Erwartungswerte beschränken. Dies würde bereits erlauben, formal anspruchsvolle Aufgaben mit geringem mathematischen Aufwand zu bewältigen.

Wie soll es nun aber gelingen, auf der Grundlage der genannten Prinzipien und Leitlinien sowie den erwähnten Rechenregeln für bedingte Erwartungswerte neue Erkenntnisse zu gewinnen? Exemplarisch soll dies am Fall der eingangs erwähnten buchwertorientierten Finanzierung skizziert werden. Dazu muss man sich klarmachen, dass die traditionelle Literatur zur Unternehmensbewertung diese Finanzierungsform bisher mit keinem Wort erwähnt hat und demzufolge auch nicht den Versuch unternahm, den Wertbeitrag der Steuervorteile bei einer festgelegten Kapitalstruktur zu Buchwerten zu ermitteln.

\footnotetext{
${ }^{20}$ Es kann auch gezeigt werden, dass dieser Zusammenhang sofort zusammenbricht, wenn man von der Voraussetzung der schwachen Auto-Regression abweicht. Siehe dazu Laitenberger/Löffler:2002.

${ }^{21}$ Sehr schön kann man dies in der Monografie Christensen/Feltham:2003 nachlesen.
} 
Es wird typischerweise unterstellt, dass sowohl der Steuersatz $\tau$ als auch der risikolose Zins $r_{f}$ in der Zukunft sichere (und der Einfachheit halber auch konstante) Größen sind. Dann ergibt sich aus einer Fremdfinanzierung in Höhe von $B_{t}^{0}$ in der folgenden Periode ein Steuervorteil in Höhe von

$$
\tau r_{f} D_{t}
$$

Es ist sofort erkennbar, dass der Steuervorteil nur eine einzige unsichere Komponente enthält: die Höhe des zukünftigen Fremdkapitals. Ist dieses unsicher, dann sind auch die zukünftigen Zinszahlungen unsicher und demzufolge auch die Steuervorteile aus der Fremdfinanzierung. Sollen diese Steuervorteile bewertet werden, so kann man den allgemein gültigen Fundamentalsatz der Preistheorie verwenden. Danach bestimmt sich der Wert einer beliebigen Zahlung durch den diskontierten Erwartungswert unter der risikoneutralen Wahrscheinlichkeit $Q$. In unserem Fall ist also der Wertbeitrag des tax shields, oder der Wertunterschied zwischen verschuldetem Unternehmen $V_{0}^{l}$ und unverschuldetem Unternehmen $V_{0}^{u}$ genau

$$
V_{0}^{l}-V_{0}^{u}=\frac{\tau r_{f} E_{Q}\left[D_{0}\right]}{1+r_{f}}+\frac{\tau r_{f} E_{Q}\left[B_{1}^{0}\right]}{\left(1+r_{f}\right)^{2}}+\mathrm{L}+\frac{\tau r_{f} E_{Q}\left[B_{T-1}^{o}\right]}{\left(1+r_{f}\right)^{T}}
$$

Vorstehende Gleichung ist ganz allgemeiner Natur. Sie gilt für jede beliebige Finanzierungspolitik. Da wir ohne weitere Voraussetzungen nichts über die risikoneutralen Wahrscheinlichkeiten sagen können, ist sie aber ebenso "schön” wie nutzlos.

Leistungsfähigkeit erlangt dieses Konzept erst dann, wenn es gelingt, zwei Dinge miteinander zu verknüpfen. Zum einen muss versucht werden, einen linearen Zusammenhang zwischen den künftigen Fremdkapitalbeständen $\stackrel{D}{t}_{t}$ und den Cashflows des Unternehmens $\mathfrak{F}_{C F}$ herzustellen. $^{22}$ Damit ist die Bewertung des tax shields auf eine Bestimmung von Erwartungswerten zukünftiger Cashflows unter $Q$ zurückgeführt. Die Bewertungsgleichung nimmt unter Berücksichtigung der Tatsache, dass der heutige Fremdkapitalbestand bekannt ist, die Form

$$
V_{0}^{l}=V_{0}^{u}+\frac{\tau r_{f} D_{0}}{1+r_{f}}+\frac{\tau r_{f} x_{1} E_{Q}\left[\tilde{F} C F_{1}\right]}{\left(1+r_{f}\right)^{2}}+\mathrm{L}+\frac{\tau r_{f} x_{T-1} E_{Q}\left[\mathcal{F} C F_{T-1}\right]}{\left(1+r_{f}\right)^{T}} .
$$

an, wobei wir hier auf eine genaue Darstellung der Parameter $x_{t}$ verzichten wollen. Dieser Erwartungswert zukünftiger Cashflows unter $Q$ ist aber immer noch eine Größe, die wir nicht berechnen können, solange wir das Wahrscheinlichkeitsmaß $Q$ nicht kennen. Da wir aber zum zweiten wissen, dass sich unsere Kapitalkosten als Diskontierungssätze eignen, haben wir zumindest prinzipiell einen Weg gefunden, diese Größen auch als Erwartungswerte unter der subjektiven Wahrscheinlichkeit des Investors darzustellen. Mit Hilfe der Gleichung (4) können wir endlich $Q$ eliminieren und wir erhalten eine Bewertungsgleichung, die sich ausschließlich auf bekannte Größen (subjektiv erwartete Cashflows, Kapitalkosten des unverschuldeten Unternehmens, Steuer- und Zinssatz) stützt.

Leider sind die Gleichungen, die sich bei entsprechender Vorgehensweise im Fall der buchwertorientierten Finanzierung ergeben, nicht gerade übersichtlich. ${ }^{23}$ Auch lassen sie sich nicht ohne weiteres elegant interpretieren. Beides jedoch stellt in unseren Augen keinen gravierenden Nachteil dar: "Interpretierbarkeit” ist keine wissenschaftliche, sondern eine subjektiv empfundene Kategorie. Wir finden es wichtiger, den Unterschied in den Wertbeiträgen zwischen buchwertorientierten und marktwertorientierter Politik exakt zu

\footnotetext{
${ }^{22}$ Dieser Zusammenhang lässt sich mit zwei Annahmen herstellen: Zum einen wäre zu unterstellen, dass das Investitionsvolumen in einem festen Verhältnis zum freien Cashflow des Unternehmens steht. Zum anderen müsste angenommen werden, dass Abweichungen zwischen Auszahlungen und Aufwendungen einzig und allein auf Investitionsauszahlungen und Abschreibungen zurückzuführen sind und die Abschreibungen linear vorgenommen werden. ${ }^{23}$ Vergleiche dazu auch Essler/Kruschwitz/Löffler:2004.
} 
beschreiben, als interpretierbare Gleichungen dort zu suchen, wo sie (leider) nicht zu finden sind.

Mit der hier beschriebenen Methode ist man auch dazu in der Lage, Unternehmen unter vielen weiteren Finanzierungspolitiken zu bewerten. Immer ist die Vorgehensweise identisch: zuerst müssen die zukünftigen Fremdkapitalbestände $B_{t}^{0}$ in ein lineares Verhältnis zu den zukünftigen Cashflows gebracht werden. Danach können die Erwartungswerte dieser Cashflows bewertet werden, weil die Kapitalkosten auch Diskontierungssätze sind. Die Grenzen unseres Ansatzes sind ebenfalls offensichtlich: immer dann, wenn es nicht gelingt, eine lineare Beziehung herzustellen, versagt das Konzept. Und eine solche Situation ist auch leicht vorstellbar. Man denke etwa an den Fall, bei dem die zukünftigen Investitionen eines Unternehmens einem von den Cashflows unabhängigen Zufallsprozess folgen und bei dem die Manager sich darauf festlegen, die Investitionen ausschließlich fremd zu finanzieren. Dann dürften sich die zukünftigen Fremdkapitalbestände nicht mehr linear in den Cashflows abbilden lassen. Und unser Ansatz führt nicht zu einem Resultat, bei dem sich das tax shield nur mit den Kapitalkosten und subjektiven Erwartungswerten der Cashflows bestimmen lässt.

\section{Zusammenfassung}

Wir stellen einen neuen Zugang zur Theorie des Discounted Cashflow vor. Unser Ansatz verknüpft das aus der Optionspreistheorie bekannte Konzept der risikoneutralen Wahrscheinlichkeit mit Fragestellungen der Unternehmensbewertung. Auf diese Weise lässt sich der in der Literatur bisher nur vage präsentierte Begriff der Kapitalkosten präzisieren und man kann Gleichungen entwickeln, mit denen sich Unternehmen vor dem Hintergrund zahlreicher bisher nicht diskutierter Finanzierungspolitiken bewerten lassen. Um den Zugang fruchtbar zu machen, ist es notwendig, eine Reihe von Annahmen zu treffen, die nicht ohne weiteres verallgemeinert werden können und die bisher auch noch nicht hinreichend in Bezug auf ihre empirische Relevanz geprüft wurden. Es bleibt eine wichtige Aufgabe zu untersuchen, inwieweit sich unsere Überlegungen auch auf Fälle übertragen lassen, in denen diese einschränkenden Annahmen nicht oder nur teilweise zutreffen.

\section{Literatur}

[1] Amram, Martha und Kulatilaka, Nalin: 1999, Real Options: Managing Strategic Investment in an Uncertain World, Harvard Business School Press, Boston, Mass.

[2] Back, Kerry und Pliska, Stanley R.: 1991, On the fundamental theorem of asset pricing with an infinite state space”, Journal of Mathematical Economics, 20, 1-18.

[3] Ballwieser, Wolfgang: 2004, Unternehmensbewertung: Prozeß, Methoden und Probleme, Schäffer-Poeschel, Stuttgart.

[4] Bernoulli, Daniel: 1738, Specimen theoriae novae de mensura sortis, Commentarii Academiae Scientiarum Imperialis Petropolitanae, 175-192, (Deutsche Übersetzung von Lutz und Peter Kruschwitz: Entwurf einer neuen Theorie zur Bewertung von Lotterien, Die Betriebswirtschaft, 56, 1996, 733-742, Englische Übersetzung von Louise Sommer: Exposition of a new theory on the measurement of risk, Econometrica, 22, 1954, 23-36).

[5] Brealey, Richard A. und Myers, Stewart C.: 2000, Principles of Corporate Finance, 6. Auflage, McGraw-Hill, New York.

[6] Canefield, Dominica: 1999, Some remarks on the valuation of firms. The Journal of Valuation, 4, 23-25.

[7] Christensen, Peter O. und Feltham, Gerald A.: 2003, Economics of Accounting, Vol. I: 
Information in Markets, Kluwer, Boston, Dordrecht, London.

[8] Copeland, Thomas E. und Antikarov, Vladimir: 2000, Real Options: A Practitioners Guide, Texere, New York, London.

[9] Copeland, Thomas E., Koller, Tim und Murrin, Jack: 2000, Valuation: Measuring and Managing the Value of Companies, John Wiley \& Sons, New York, 3. te Auflage.

[10] Copeland, Thomas E. und Weston, J. Fred: 1988, Financial Theory and Corporate Policy, 3. Auflage, Addison-Wesley, Reading, Mass.

[11] Diedrich, Rolf: 2003, Die Sicherheitsäquivalentmethode der Unternehmensbewertung: ein (auch) entscheidungstheoretisch wohlbegründbares Verfahren - Anmerkungen zu dem Beitrag von Wolfgang Kürsten in der zfbf, Zeitschrift für betriebswirtschaftliche Forschung, 55, 281-286.

[12] Drukarczyk, Jochen: 2003, Unternehmensbewertung, 4. edn, Vahlen, München.

[13] Essler, Wolfgang, Kruschwitz, Lutz und Löffler, Andreas: 2004, Zur Anwendung des WACC-Verfahrens bei vorgegebener bilanzieller Verschuldung, Betriebswirtschaftliche Forschung und Praxis, 2/2004, 133-147.

[14] Feltham, Gerald A. und Ohlson, James A.: 1995, Valuation and clean surplus accounting for operating and financial activities, Contemporary Accounting Research, 11, 689-731.

[15] Garman, Mark B. und Ohlson, James A.: 1981, Valuation of risky assets in arbitragefree economies with transaction costs, Journal of Financial Economics, 9, 271-280.

[16] Hachmeister, Dirk: 2000, Der Discounted Cash Flow als Maß der Unternehmenswertsteigerung, 4. edn, Lang, Frankfurt am Main.

[17] Harris, Robert und Pringle, John: 1985, Risk-adjusted discount rates - extension from the average-risk case, Journal of Financial Research, 8, 237-244.

[18] Hering, Thomas: 1999, Finanzwirtschaftliche Unternehmensbewertung, Deutscher Universitäts-Verlag, Wiesbaden.

[19] Hommel, Ullrich, Scholich, Martin und Vollrath, Robert (eds): 2001, Realoptionen in der Unternehmenspraxis: Wert schaffen durch Flexibilität, Springer, Berlin.

[20] Ingersoll, Jonathan E.: 1987, Theory of Financial Decision Making. Rowman \& Littlefield, Totowa, NJ.

[21] Institut der Wirtschaftsprüfer in Deutschland, Herausgeber, 2002: Handbuch für Rechnungslegung, Prüfung und Beratung, Band II. IdW-Verlag, Düsseldorf, 12. Auflage.

[22] Koch, Christian: 1999, Optionsbasierte Unternehmensbewertung. Realoptionen im Rahmen von Akquisitionen, Deutscher Universitäts-Verlag, Wiesbaden.

[23] Kruschwitz, Lutz und Löffler, Andreas: 2003a, DCF (Part I), available on www.ssrn.com, paper-ID 389408.

[24] Kruschwitz, Lutz und Löffler, Andreas: 2003b, Semi-subjektive Bewertung, Zeitschrift für Betriebswirtschaft, 73, 1335-1345.

[25] Kruschwitz, Lutz und Löffler, Andreas: 2004, Bemerkungen über Kapitalkosten vor und nach Steuern, Zeitschrift für Betriebswirtschaft, 74, 1075-1090.

[26] Kürsten, Wolfgang: 2002, 'Unternehmensbewertung unter Unsicherheit' oder: Theoriedefizit einer künstlichen Diskussion über Sicherheitsäquivalent und Risikozuschlagsmethode. - Anmerkungen (nicht nur) zu dem Beitrag von Bernhard 
Schwetzler in der zfbf, Zeitschrift für betriebswirtschaftliche Forschung, 54, 128-144.

[27] Kürsten, Wolfgang: 2003, Grenzen und Reformbedarfe der Sicherheitsäquivalentmethode in der (traditionellen) Unternehmensbewertung: Erwiderung auf die Anmerkungen von Ralf Diedrich und Jörg Wiese in der zfbf, Zeitschrift für betriebswirtschaftliche Forschung, 55, 306-314.

[28] Laitenberger, Jörg: 2004, Rendite und Kapitalkosten, Diskussionspapier Nr. 295, Wirtschaftwissenschaftliche Fakultät der Universität Hannover.

[29] Laitenberger, Jörg und Löffler, Andreas: 2002, Capital budgeting in arbitrage free markets, Diskussionspapier Nr. 258, Wirtschaftwissenschaftliche Fakultät der Universität Hannover.

[30] Löffler, Andreas: 2004, Zwei Anmerkungen zu WACC, Zeitschrift für Betriebswirtschaft, 74, 933-942.

[31] Mandl, Gerwald und Rabel, Klaus: 1997, Unternehmensbewertung, Ueberreuter, Wien, Frankfurt am Main.

[32] de Matos, Joao Amaro: 2001, Theoretical Foundations of Corporate Finance, Princeton University Press, Princeton, NJ.

[33] Miles, James A. und Ezzell, John R.: 1980, The weighted average cost of capital, perfect capital markets, and project life: a clarification, Journal of Financial and Quantitative Analysis, 15, 719-730.

[34] Musiela, Marek und Rutkowski, Marek: 1998, Martingale Methods in Financial Modelling, $2^{\text {nd }}$ ed., Springer, Berlin.

[35] Nowak, Karsten: 2003, Unternehmensbewertung: Discounted Cash Flow, Realoption, Economic Value Added und der Direct Comparison Approach, 2. edn, Deutscher Universitäts-Verlag, Wiesbaden.

[36] Peemöller, Volker H. (ed.): 2001, Praxishandbuch der Unternehmensbewertung, Neue Wirtschafts-Briefe, Herne, Berlin.

[37] Richter, Frank: 2002, Kapitalmarktorientierte Unternehmensbewertung: Konzeption, finanzwirtschaftliche Bewertungsprämissen und Anwendungsbeispiel, Lang, Frankfurt am Main.

[38] Ross, Stephen A.: 1978, A Simple Approach to the Valuation of Risky Streams, Journal of Business, 51, 453-475.

[39] Schildbach, Thomas: 2000, Ein fast problemloses Dcf-Verfahren zur Unternehmensbewertung, Zeitschrift für betriebswirtschaftliche Forschung, 52, 707-723.

[40] Schwetzler, Bernhard: 2000, Unternehmensbewertung unter Unsicherheit: Sicherheitsäquivalent- $\quad$ oder $\quad$ Risikozuschlagsmethode?, Zeitschrift für betriebswirtschaftliche Forschung, 52, 469-486.

[41] Schwetzler, Bernhard: 2002, Das Ende des Ertragswertverfahrens? Replik zu den Anmerkungen von Wolfgang Kürsten zu meinem Beitrag in der zfbf, Zeitschrift für betriebswirtschaftliche Forschung, 54, 145-158.

[42] Spremann, Klaus: 2002, Finanzanalyse und Unternehmensbewertung, Oldenbourg, München, Wien.

[43] Stehle, R.: 2004, Die Festlegung der Risikoprämie von Aktien im Rahmen der Schätzung des Wertes von börsennotierten Kapitalgesellschaften, Die 
Wirtschaftsprüfung, 57, 906-927.

[44] Taggart, Robert A. jr.: 1991, 'Consistent valuation and cost of capital expressions with corporate and personal taxes', Financial Management, 20, 8-20.

[45] Tomaszewski, Claude: 2000, Bewertung strategischer Flexibilität beim Unternehmenserwerb: Der Wertbeitrag von Realoptionen, Lang, Frankfurt am Main.

[46] Trigeorgis, Lenos G.: 1986, Valuing Real Investment Opportunities: An Options Approach to Strategic Capital Budgeting, PhD thesis, Harvard University.

[47] Wiese, Jörg: 2003, Zur theoretischen Fundierung der Sicherheitsäquivalentmethode und des Begriffs der Risikoauflösung bei der Unternehmensbewertung: Anmerkungen zu dem Beitrag von Wolfgang Kürsten in der zfbf, Zeitschrift für betriebswirtschaftliche Forschung, 55, 287-305.

[48] Wilhelm, Jochen: 2004, Unternehmensbewertung bei persönlicher Einkommensteuer: Sind die Kapitalkosten ein fruchtbares Konzept? In H. Wildemann (ed.), Organisation und Personal: Festschrift für Rolf Bühner, TCW Transfer-Centrum, München, 941-961. 